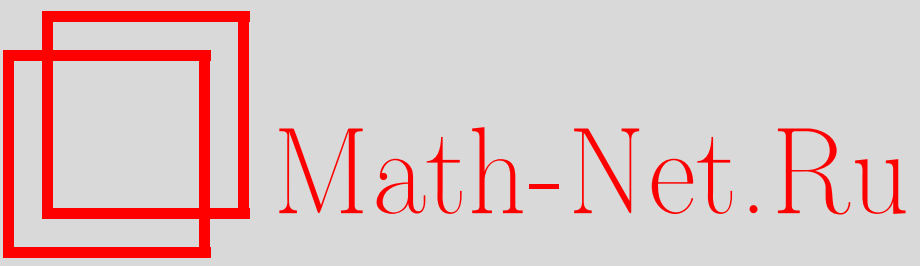

Н. Г. Мусакаев, М. К. Хасанов, С. Л. Бородин, Построение аналитического решения задачи об образовании газового гидрата в пористом пласте, Итоги науки и техн. Сер. Соврем. мат. и ее прил. Темат. обз., 2019, том 172, 91-95

DOI: https://doi.org/10.36535/0233-6723-2019-172-91-95

Использование Общероссийского математического портала Math-Net.Ru подразумевает, что вы прочитали и согласны с пользовательским соглашением

http://www.mathnet.ru/rus/agreement

Параметры загрузки:

IP: 54.198 .55 .26

26 апреля 2023 г., 15:37:15 


\title{
ПОСТРОЕНИЕ АНАЛИТИЧЕСКОГО РЕШЕНИЯ ЗАДАЧИ ОБ ОБРАЗОВАНИИ ГАЗОВОГО ГИДРАТА В ПОРИСТОМ ПЛАСТЕ
}

\author{
(c) 2019 г \\ Н. Г. МУСАКАЕВ, М. К. ХАСАНОВ, С. Л. БОРОДИН
}

\begin{abstract}
АннотАция. Приведена математическая постановка задачи о закачке холодного газа в пористую среду, сопровождающейся образованием газогидрата, в форме начально-краевой задачи для системы нелинейных дифференциальных уравнений в частных производных, выражающих законы сохранения массы и энергии, и дополненных условиями баланса массы и тепла на подвижной границе фазовых переходов. В прямолинейно-параллельном приближении построены приближенные аналитические решения со скачком температуры на границе образования газового гидрата, описывающие зависимость между координатой границы фазового перехода и параметрами закачиваемого газа и пористой среды.
\end{abstract}

Ключевые слова: дифференциальное уравнение, математическая модель, аналитические решения, фильтрация, газовый гидрат, фазовый переход.

\section{CONSTRUCTION OF AN ANALYTICAL SOLUTION OF THE PROBLEM ON THE FORMATION OF GAS HYDRATE IN A POROUS MINE}

\author{
(c) 2019 N. G. MUSAKAEV, M. K. KHASANOV, S. L. BORODIN
}

\begin{abstract}
In this paper, we propose the mathematical formulation of the problem on injection of a cold gas into a porous medium accompanied by the formation of gas hydrate. The mathematical formulation of the problem is an initial-boundary-value problem for a system of nonlinear partial differential equations expressing the laws of conservation of mass and energy supplemented by the conditions of mass and heat balance at the moving boundary of phase transitions. Within the framework of the rectilinear parallel approximation, we construct approximate analytical solutions with a temperature jump at the boundary where the gas hydrate appears. These solutions describe the relationship between the coordinate of the boundary of phase transition and the parameters of the injected gas and porous medium.
\end{abstract}

Keywords and phrases: differential equation, mathematical model, analytical solutions, filtration, gas hydrate, phase transition.

AMS Subject Classification: 35C06, 76S05, 76N15, 82B26

1. Введение. В настоящее время широко используемым и удобным источником энергии является природный газ (см. [9]). Во многом это связано с весьма высокой эффективностью выработки электроэнергии, большой теплотой сгорания и низким уровнем выбросов углерода (по сравнению с углем и нефтью). Поэтому с экологической точки зрения природный газ является одним из самых безопасных видов органического топлива (см. [12]).

Работа выполнена при поддержке Российского фонда фуедаментальных исследований (проект № 18-29-10023). 
Ряд исследователей указывает на увеличение в течение ближайших двадцати лет доли природного газа в энергетике. В этой связи во всем мире возрастает потребность в разработке технологий для эффективного хранения углеводородного газа (см. [12]). Одним из способов хранения природного газа является его консервация в газогидратной форме в пористых пластах (см. [1,2,8,11]). Подземная консервация в истощенных коллекторах месторождений углеводородного сырья и/или водоносных горизонтах является достаточно распространенным методом хранения природного газа. Преимуществами газогидратной формы хранения углеводородного газа в пористых пластах являются: достаточно компактный характер хранения, так как в одном и том же объеме при одинаковых термодинамических условиях в газовом гидрате присутствует значительно больше газа, чем в свободном состоянии; относительно умеренные по давлению и температуре условия формирования и хранения; невзрывоопасный характер хранения (см. [1,8,12]).

Изучению процесса образования газовых гидратов в пористом пласте посвящены работы $[4,6,7,10]$. Предлагаемое исследование отличается тем, что в настоящей работе построены аналитические решения задачи в прямолинейно-параллельном приближении и проведен анализ этих решений.

2. Решение с непрерывным распределением температуры. Рассмотрим следующую постановку задачи. Пусть имеется горизонтальный полубесконечный пористый пласт, насыщенный в начальном состоянии газом (метаном) и водой. Через левую границу пласта $(x=0)$ под постоянным давлением $p_{e}$ нагнетается метан с известной и постоянной температурой $T_{e}$, причем значения $p_{e}$ и $T_{e}$ таковы, что в пористой среде может происходить формирование газогидрата. Рассмотрим случай, когда образование газового гидрата полностью происходит на фронтальной границе, т.е. в пласте возникают две области. В первой (ближней к границе нагнетания, $x=0$ ) зоне вода полностью перешла в газогидратное состояние, поэтому в порах присутствуют только газ и гидрат $\mathrm{CH}_{4}$. Во второй области пористая среда насыщена газом и водой. На поверхности $x=x_{(s)}$, разделяющей две выделенные зоны, гидратонасыщенность $S_{h}$ меняется скачком от $S_{h}^{-}=S_{h(1)}$ до $S_{h}^{+}=0$.

Для такой постановки задачи в $[5,10]$ на основе методов и подходов механики многофазных сред (см. [3]) записана система нелинейных дифференциальных уравнений, описывающая течение газа в пористой среде с учетом образования газового гидрата. Для введенной автомодельной переменной $\xi=x / \sqrt{\chi^{(T)} t}\left(\chi^{(T)}\right.$ - коэффициент температуропроводности пласта, $t$ - время) данная система на основе метода линеаризации Лейбензона представлена в виде системы обыкновенных дифференциальных уравнений второго порядка (уравнения температуро- и пьезопроводности):

$$
-\frac{\xi}{2} \frac{d p_{(i)}^{2}}{d \xi}=\eta_{(i)} \frac{d}{d \xi}\left(\frac{d p_{(i)}^{2}}{d \xi}\right), \quad-\frac{\xi}{2} \frac{d T_{(i)}}{d \xi}=\frac{P e_{(i)}}{2 p_{0}^{2}} \frac{d p_{(i)}^{2}}{d \xi} \frac{d T_{(i)}}{d \xi}+\frac{d}{d \xi}\left(\frac{d T_{(i)}}{d \xi}\right)
$$

где

$$
\begin{aligned}
\eta_{(i)}=\frac{\chi_{(i)}^{(P)}}{\chi^{(T)}}, \quad \chi_{(i)}^{(P)} & =\frac{k_{(i)} p_{0}}{m S_{g(i)} \mu_{g}}, \quad P e_{(i)}=\frac{\rho_{g 0} p_{0} c_{g} k_{(i)}}{\lambda \mu_{g}}, \quad k_{(i)}=k_{0} S_{g(i)}^{3}, \quad i=1,2, \\
\lambda & =(1-m) \lambda_{s k}+m \sum_{j=g, l, h} S_{j} \lambda_{j}, \quad \sum_{j=g, l, h} S_{j}=1 .
\end{aligned}
$$

Здесь параметры первой и второй зон снабжены нижними индексами в скобках $i=1,2$; нижние индексы $s k, h, l$ и $g$ относятся к параметрам пористого скелета, гидрата, воды и газа соответственно; $p_{0}$ и $T_{0}$ - исходные давление и температура в пласте; $\rho_{j}, c_{j}$ и $\lambda_{j}(j=s k, h, l$ и $g)$ - истинная плотность, удельная теплоемкость и коэффициент теплопроводности $j$-й фазы; $S_{j}(j=g, l, h)-$ насыщенность пор $j$-й фазой; $m$ и $k_{0}$ - пористость и абсолютная проницаемость пласта; $\mu_{g}-$ динамическая вязкость газовой фазы.

Для распределения давления и температуры в каждой из выделенных областей могут быть записаны следующие соотношения: 


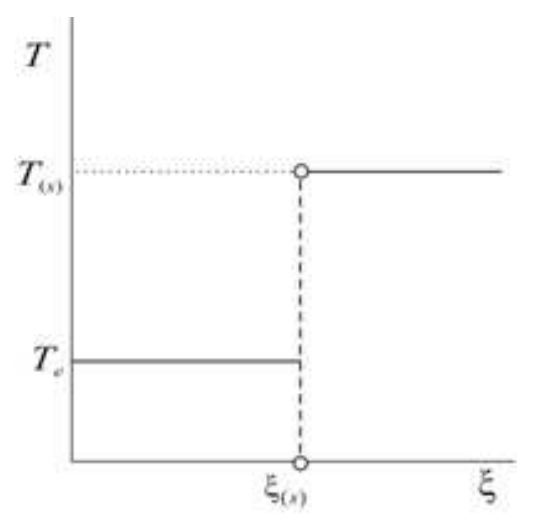

Рис. 1. Схематическое распределение температуры в пласте

$$
\begin{array}{ll}
p_{(1)}^{2}=p_{(s)}^{2}+\frac{\left(p_{e}^{2}-p_{(s)}^{2}\right) \int_{\xi}^{\xi_{(s)}} \exp \left(-\frac{\xi^{2}}{4 \eta_{(1)}}\right) d \xi}{\int_{0}^{\xi_{(s)}} \exp \left(-\frac{\xi^{2}}{4 \eta(1)}\right) d \xi}, & 0<\xi<\xi_{(s)}, \\
T_{(1)}=T_{(s)}+\frac{\left(T_{e}-T_{(s)}\right) \int_{\xi}^{\xi(s)} \exp \left(-\frac{\xi^{2}}{4}-\frac{P e_{(1)}}{2 p_{0}^{2}} p_{(1)}^{2}\right) d \xi}{\int_{0}^{\xi_{(s)}} \exp \left(-\frac{\xi^{2}}{4}-\frac{P e_{(1)}}{2 p_{0}^{2}} p_{(1)}^{2}\right) d \xi}, & 0<\xi<\xi_{(s)}, \\
p_{(2)}^{2}=p_{0}^{2}+\frac{\left(p_{(s)}^{2}-p_{0}^{2}\right) \int_{\xi}^{\infty} \exp \left(-\frac{\xi^{2}}{4 \eta_{(2)}}\right) d \xi}{\int_{\xi_{(s)}}^{\infty} \exp \left(-\frac{\xi^{2}}{4 \eta_{(2)}}\right) d \xi}, & \xi_{(s)}<\xi<\infty, \\
T_{(2)}=T_{0}+\frac{\left(T_{(s)}-T_{0}\right) \int_{\xi}^{\infty} \exp \left(-\frac{\xi^{2}}{4}-\frac{P e_{(2)}}{2 p_{0}^{2}} p_{(2)}^{2}\right) d \xi}{\int_{\xi_{(s)}}^{\infty} \exp \left(-\frac{\xi^{2}}{4}-\frac{P e_{(2)}}{2 p_{0}^{2}} p_{(2)}^{2}\right) d \xi} & \xi_{(s)}<\xi<\infty,
\end{array}
$$

где нижний индекс $s$ в скобках относится к параметрам на границе гидратообразования.

Построенные решения (2), (3) описывают распределения давления и температуры в отдельных областях пласта. При подстановке этих решений в соотношения на границах фазовых переходов возникает система трансцендентных уравнений, которая в общем случае может быть решена только с использованием численных методов. Однако принципиальный интерес представляет получение аналитического решения, устанавливающего определенный вид функциональной зависимости координаты границы фазового перехода от параметров закачиваемого газа и пористой среды. Данное решение является удобным инструментом для анализа математической модели образования газогидрата в пористой среде.

3. Решение со скачком температуры на границе фазовых переходов. При построении приближенного аналитического решения используем результаты расчетов, которые показывают, что распределение температуры в пласте близко к ступенчатому со скачком температуры на фронте образования газового гидрата (см. [5]). В этом случае поле температуры в окрестностях границы фазовых переходов определяется двумя значениями: температура нагнетания метана $T_{e}$ и равновесная температура гидратообразования $T_{(s)}$ (рис. 1$)$.

Согласно проведенным расчетам распределение температуры в пласте близко к ступенчатому при высоких значениях проницаемости пористой среды. В этом случае поглощение газа на границе гидратообразования оказывает малое влияние на изменение давления в пласте. Поэтому в уравнении баланса массы на границе гидратообразования можно пренебречь слагаемым, отвечающим за поглощение газа. Тогда с учетом ступенчатого распределения температуры в пласте 
условия баланса массы и тепла на фронте гидратообразования могут быть представлены в следующем виде (см. [3, 10]):

$$
\begin{gathered}
m S_{g(2)} \rho_{g(s)}\left(v_{g(2)}-\dot{x}_{(s)}\right)=m S_{g(1)} \rho_{g(s)}\left(v_{g(1)}-\dot{x}_{(s)}\right), \\
m S_{g(1)} \rho_{g(1)}\left(v_{g(1)}-\dot{x}_{(s)}\right) c_{g} T_{e}+m S_{h(1)} \rho_{h} L_{h} \dot{x}_{(s)}+\rho c\left(T_{(s)}-T_{e}\right) \dot{x}_{(s)}= \\
=m S_{g(2)} \rho_{g(2)}\left(v_{g(2)}-\dot{x}_{(s)}\right) c_{g} T_{(s)}, \\
T_{(s)}=T_{0}+T_{*} \ln \frac{p_{(s)}}{p_{s 0}}, \quad \rho c=(1-m) \rho_{s k} c_{s k}+m \sum_{j=g, l, h} S_{j} \rho_{j} c_{j},
\end{gathered}
$$

где $v_{g}$-скорость газовой фазы; $\dot{x}_{(s)}$-скорость движения границы фазового перехода; $L_{h}-$ удельная теплота гидратообразования; $p_{s 0}$ - равновесное давление, соответствующее исходной температуре $T_{0} ; T_{*}$ - эмпирический параметр, имеющий размерность температуры и зависящий от состава природного газа.

Уравнения (4) и (5) с учетом закона Дарси и уравнения состояния калорически совершенного газа примут вид:

$$
\begin{gathered}
\frac{k_{(2)}}{T_{(s)}} \frac{d p_{(2)}^{2}}{d x}-\frac{k_{(1)}}{T_{e}} \frac{d p_{(1)}^{2}}{d x}=2 m \mu_{g}\left(S_{g(1)} \frac{p_{(s)}}{T_{e}}-S_{g(2)} \frac{p_{(s)}}{T_{(s)}}\right) \dot{x}_{(s)}, \\
k_{(1)} \frac{d p_{(1)}^{2}}{d x}-k_{(2)} \frac{d p_{(2)}^{2}}{d x}=2 \mu_{g} \frac{R_{g}}{c_{g}}\left(\rho c\left(T_{(s)}-T_{e}\right)+m S_{h(1)} \rho_{h} L_{h}+m c_{g} \frac{p_{(s)}}{R_{g}}\left(S_{g(2)}-S_{g(1)}\right)\right) \dot{x}_{(s)} ;
\end{gathered}
$$

здесь $R_{g}$ - газовая постоянная. Для автомодельной переменной $\xi$ уравнения $(7)$ и $(8)$ могут быть переписаны в следующем виде:

$$
\begin{gathered}
\frac{k_{(2)}}{T_{(s)}} \frac{d p_{(2)}^{2}}{d \xi}-\frac{k_{(1)}}{T_{e}} \frac{d p_{(1)}^{2}}{d \xi}=m \mu_{g} \chi^{(T)}\left(S_{g(1)} \frac{p_{(s)}}{T_{e}}-S_{g(2)} \frac{p_{(s)}}{T_{(s)}}\right) \xi_{(s)}, \\
k_{(1)} \frac{d p_{(1)}^{2}}{d \xi}-k_{(2)} \frac{d p_{(2)}^{2}}{d \xi}=\mu_{g} \frac{R_{g}}{c_{g}}\left(\rho c\left(T_{(s)}-T_{e}\right)+m S_{h(1)} \rho_{h} L_{h}+m c_{g} \frac{p_{(s)}}{R_{g}}\left(S_{g(2)}-S_{g(1)}\right)\right) \chi^{(T)} \xi_{(s)} .
\end{gathered}
$$

Умножив соотношение $(9)$ на $T_{(s)}$ и сложив с $(10)$, получим

$$
k_{(1)}\left(1-\frac{T_{(s)}}{T_{e}}\right) \frac{d p_{(1)}^{2}}{d \xi}=\mu_{g} R_{g}\left(m S_{h(1)} \rho_{h} \frac{L_{h}}{c_{g}}-m S_{g(1)} \frac{p_{(s)}}{R_{g}}\left(1-\frac{T_{(s)}}{T_{e}}\right)+\frac{\rho c}{c_{g}}\left(T_{(s)}-T_{e}\right)\right) \chi^{(T)} \xi_{(s)} .
$$

Из этого уравнения для автомодельной координаты границы образования газогидрата $\xi_{(s)}$ можно записать

$$
\xi_{(s)}=\frac{k_{(1)}}{\mu_{g} R_{g} \chi^{(T)}}\left(m S_{h(1)} \rho_{h} T_{e} \frac{L_{h}}{c_{g}\left(T_{(s)}-T_{e}\right)}-m S_{g(1)} \frac{p_{(s)}}{R_{g}}-\frac{\rho c T_{e}}{c_{g}}\right)^{-1} \frac{d p_{(1)}^{2}}{d \xi} .
$$

Будем пренебрегать разностью коэффициента проницаемости в двух зонах пласта и поглощением газа на границе фазовых переходов. Тогда из первого уравнения системы (1) можно получить приближенное решение для распределения давления в первой области:

$$
p_{(1)}^{2}=p_{e}^{2}+\frac{p_{0}^{2}-p_{e}^{2}}{\sqrt{\pi \eta_{(1)}}} \int_{0}^{\xi} \exp \left(-\frac{\xi^{2}}{4 \eta_{(1)}}\right) d \xi \quad \text { или } \quad \frac{d p_{(1)}^{2}}{d \xi}=\frac{p_{0}^{2}-p_{e}^{2}}{\sqrt{\pi \eta_{(1)}}} \exp \left(-\frac{\xi^{2}}{4 \eta_{(1)}}\right) .
$$

Поскольку для высокопроницаемых пористых сред $\eta_{(1)} \gg \xi^{2}$, то из (12) получим:

$$
\frac{d p_{(1)}^{2}}{d \xi}=\frac{p_{0}^{2}-p_{e}^{2}}{\sqrt{\pi \eta_{(1)}}}, \quad p_{(s)}=p_{e}
$$


Подставляя (13) в соотношение (11), для автомодельной координаты границы образования газогидрата можно записать

$$
\xi_{(s)}=\frac{k_{(1)}}{\mu_{g} R_{g} \chi^{(T)}}\left(m S_{h(1)} \rho_{h} T_{e} \frac{L_{h}}{c_{g}\left(T_{(s)}-T_{e}\right)}-m S_{g(1)} \frac{p_{(s)}}{R_{g}}-\frac{\rho c T_{e}}{c_{g}}\right)^{-1} \frac{p_{0}^{2}-p_{e}^{2}}{\sqrt{\pi \eta_{(1)}}}
$$

или с учетом (5) и (13)

$$
\xi_{(s)}=\frac{k_{(1)} c_{g}\left(p_{0}^{2}-p_{e}^{2}\right)}{\mu_{g} R_{g} \chi^{(T)} \sqrt{\pi \eta(1)}}\left(\rho c T_{e}-m S_{h(1)} \rho_{h} T_{e} \frac{L_{h}}{T_{e}-T_{0}-T_{*} \ln \left(p_{e} / p_{s 0}\right)}+m S_{g(1)} c_{g} \frac{p_{e}}{R_{g}}\right)^{-1} .
$$

Таким образом, получено приближенное аналитическое решение в виде явной функциональной зависимости координаты границы гидратообразования от давления и температуры закачиваемого газа и параметров пористой среды.

\section{СПИСОК ЛИТЕРАТУРЫ}

1. Бондарев Э. А., Рожин И. И., Попов В. В., Аргунова К. К. Оценка возможности подземного хранения гидратов природного газа в зоне многолетней мерзлоты// Криосфера Земли. - 2015. - 19, № 4. C. $64-74$.

2. Кузнецов Ф. А., Истомин В. А., Родионова Т. В. Газовые гидраты: исторический экскурс, современное состояние, перспективы исследований// Рос. хим. ж. - 2003. - 47, № 3. - С. 5-18.

3. Нигматулин Р. И. Динамика многофазных сред. - М.: Наука, 1987.

4. Нурисламов O. Р., Шагапов B. Ш. Нагнетание газа во влажную пористую среду с образованием газогидрата// Прикл. мат. мех. - 2009. - 73, № 5. - С. 809-823.

5. Шагапов В. Ш., Мусакаев Н. Г., Хасанов М. К. Нагнетание газа в пористый резервуар, насыщенный газом и водой // Теплофиз. аэромех. - 2005. - 12, № 4. - С. 645-656.

6. Шагапов В. Ш., Хасанов М. К., Гималтдинов И. К., Столповский М. В. Численное моделирование образования газогидрата в пористом пласте конечной протяженности при продувке его газом// Прикл. мех. техн. физ. - 2011. - 52, № 4. - С. 116-126.

7. Шагапов В. Ш., Хасанов М. К., Мусакаев Н. Г. Образование газогидрата в пористом резервуаре, частично насыщенном водой, при инжекции холодного газа// Прикл. мех. техн. физ. - 2008. - 49, № 3. - C. 137-150.

8. Chatti I., Delahaye A., Fournaison L., Petitet J. P. Benefits and drawbacks of clathrate hydrates: a review of their areas of interest// Energy Conv. Manag. — 2005. — 46, № 9-10. — P. 1333-1343.

9. Demirbas A. Natural gas// in: Methane Gas Hydrate. — London: Springer, 2010. — P. 57-76.

10. Shagapov V. Sh., Musakaev N. G., Khasanov M. K. Formation of gas hydrates in a porous medium during an injection of cold gas// Int. J. Heat Mass Trans. - 2015. — 84. - P. 1030-1039.

11. Sloan E. D., Koh C. A. Natural gas hydrates: Recent advances and challenges in energy and environmental applications// AIChE J. — 2007. — 53, № 7. — P. 1636-1643.

12. Veluswamy H. P., Kumar A., Seo Y., Lee J. D., Linga P. A review of solidified natural gas (SNG) technology for gas storage via clathrate hydrates// Appl. Energy. — 2018. — 216. — P. 262-285.

Мусакаев Наиль Габсалямович

Тюменский филиал Института теоретической и прикладной механики

им. С. А. Христиановича СО РАН;

Тюменский индустриальный университет

E-mail: musakaev@ikz.ru

Хасанов Марат Камилович

Стерлитамакский филиал Башкирского государственного университета

E-mail: hasanovmk@mail.ru

Бородин Станислав Леонидович

Тюменский филиал Института теоретической и прикладной механики

им. С. А. Христиановича СО РАН

E-mail: borodin@ikz.ru 